\title{
14-3-3 sigma and p53 expression in gastric cancer and its clinical applications
}

\author{
Gilbert Mühlmann $^{\mathrm{a}, *}$, Dietmar Öfner ${ }^{\mathrm{b}}$, Matthias Zitt ${ }^{\mathrm{a}}$, Hannes M. Müller $^{\mathrm{a}}$, Hans Maier ${ }^{\mathrm{c}}$, \\ Patrizia Moser ${ }^{\mathrm{c}}$, Kurt W. Schmid ${ }^{\mathrm{d}}$, Marion Zitt ${ }^{\mathrm{a}}$ and Albert Amberger ${ }^{\mathrm{e}}$ \\ ${ }^{a}$ Center of Operative Medicine, Department of Visceral, Transplant and Thoracic Surgery, Innsbruck Medical \\ University, Austria \\ ${ }^{\mathrm{b}}$ Department of Surgery, Paracelsus Private Medical University, Salzburger Landeskliniken, Salzburg, Austria \\ ${ }^{\mathrm{c}}$ Department of Pathology, Innsbruck Medical University, Austria \\ ${ }^{\mathrm{d}}$ Institute of Pathology and Neuropathology, University Hospital Essen, Germany \\ ${ }^{\mathrm{e}}$ Tyrolean Cancer Research Institute, Innsbruck, Austria
}

\begin{abstract}
G2 arrest enabling the repair of damaged DNA. The function of 14-3-3 $\sigma$ is frequently lost in tumor cells, indicating a potential tumor suppressor function. The purpose of this study was to evaluate the prognostic value of 14-3-3 $\sigma$ expression in human gastric cancer. 14-3-3 $\sigma$ expression was analyzed by immunohistochemistry in 157 tumor samples of patients, who underwent resection for gastric cancer. Since 14-3-3 $\sigma$ is involved in the p53 network, p53 expression was detected in parallel and correlated with 14-3-3 $\sigma$. 14-3-3 $\sigma$ was found to be overexpressed in 75 (47.8\%) of 157 cases, the overexpression rate of $\mathrm{p} 53$ protein was $27.4 \%$. 14-3-3 $\sigma$ overexpression was statistically significantly associated with pT-stage $(p=0.041) \mathrm{pN}$-stage $(p=0.015)$ and UICC-stage $(p=0.019)$ and showed a borderline significance with Lauren classification $(p=0.057)$. Univariate survival calculations revealed a coexistent 14-3-3 $\sigma$ and p53 overexpression as a significant predictor of disease-free survival. Multivariate analysis did not unfold 14-3-3 as an independent prognostic factor for disease-free survival and overall survival. Concomitant 14-3-3 $\sigma$ and p53 overexpression in tumor cells of patients with gastric cancer identifies a population of patients with relatively unfavorable prognosis.
\end{abstract}

Keywords: Gastric cancer, 14-3-3 sigma, tumorigenesis

\section{Introduction}

14-3-3 proteins have a critical role in a wide variety of cellular responses including signal transduction, cell cycle regulation, apoptosis, cytoskeleton organisation and malignant transformation [1-3]. The 14-3-3 protein family comprises 7 isoforms whereas $14-3-3 \sigma$ is the only isoform induced by $\mathrm{p} 53$ protein in response to $\gamma$ irradiation and other damaging agents [4]. 14-3-3 $\sigma$ sequesters the mitotic initiation complex cdc2-cyclin

\footnotetext{
*Corresponding author: Gilbert Mühlmann, M.D., Center of Operative Medicine, Department of Visceral, Transplant and Thoracic Surgery, Innsbruck Medical University, Anichstrasse 35, A-6020 Innsbruck, Austria. Tel:: +43 512504 80616; Fax: +43 512504 28519; E-mail: gilbert.muehlmann@i-med.ac.at.
}

B1 from entering the nucleus, thus preventing initiation of mitosis. In this manner, 14-3-3 $\sigma$ induces G2 arrest enabling the repair of damaged DNA [1]. As a target gene of p53, 14-3-3 $\sigma$ has reciprocally influence on the p53 network. It specifically increase p53 stability and enhance p53 transcriptional activity [5].

The function of 14-3-3 $\sigma$ is frequently lost not only in tumor cells but also in surrounding pre-dysplastic tissue, indicating an important tumor suppressor function that becomes lost at an early stage in the progression to invasive cancer [6,7]. Besides, Wilker et al. indicate that 14-3-3 $\sigma$ is important in the physiological downregulation of new protein synthesis during and immediately after mitosis and is required for the translation of a protein kinase that is essential to the proper completion of cytokinesis [3]. The loss of function is either due 
to hypermethylation of the 14-3-3 $\sigma$ promoter or to induction of an oestrogen-responsive ubiquitin ligase that specifically targets 14-3-3 $\sigma$ for proteosomal degradation [6-12]. CpG islands hypermethylation and loss of 14-3-3 $\sigma$ expression was frequently found in different tumor entities such as hepatocellular carcinoma, lung cancer, prostate cancer, oral cancer, ovarian cancer or gastric cancer whereas in colorectal cancer $\mathrm{CpG}$ islands were unmethylated [10-16]. In the present study we aimed to explore the expression of 14-3-3 $\sigma$ in the tissue of patients with resected gastric cancer and to elucidate the relationship to biological behaviour and prognosis.

\section{Material and methods}

\subsection{Patients and specimens}

The study was conducted according to the regulations of the local ethics committee and Austrian law. Tumor tissues of 157 consecutive cases of adenocarcinomas of the stomach $\{97$ men $(62 \%)$ and 60 women $(38 \%)$ at a mean age of 67.2 years (95\% CI: 65.8-69.2) $\}$ were investigated in this study. All patients had been operated between 1993 and 2004 at the Department of Surgery, Innsbruck Medical University, Austria with curative intent ( $n=157$ ). Tumor tissues were routinely processed (formalin-fixed and paraffin-embedded) and were classified according to the guidelines of the Union Internationale Contre le Cancer (UICC) and were graded according to WHO classification [17,18]. Adjuvant or additive chemotherapy was recommended to patients with UICC stages III and IV. Adjuvant chemoradiation as propagated by Macdonald et al. was not used routinely but carried out in selected patients [19]. Demographic data of all patients were recruited from our computerised surgical documentation system (ChiBASE). All data regarding date and cause of death were confirmed by the "Tumorregister Tirol", a cancer-register maintained by the Tyrolean government. Demographic data and tumor characteristics are summarised in Table 1.

\subsection{Immunohistochemistry}

Sections of a tissue microarray were deparaffinized by prolonged incubation in xylene (3-4 min), followed by prolonged washing and rehydration in ethanol $(96 \%$ ethanol 2-3 min, $80 \%$ ethanol $3 \mathrm{~min}$, and $70 \%$ ethanol $3 \mathrm{~min}$ ). Endogenous peroxidase activity was blocked by $0.5 \%$ hydrogen peroxide $(\mathrm{H} 2 \mathrm{O} 2)$ in methanol for $20 \mathrm{~min}$. Antigen retrieval was achieved by heating the slides to $86^{\circ} \mathrm{C}$ in citrate buffer $(0.01 \mathrm{M})$ for $15 \mathrm{~min}$. Immunohistochemistry was performed as described earlier [20]. Briefly, slides were incubated with anti-14-3-3 $\sigma$ antibody (2 lg/ml, Neomarkers, Fremont, CA, USA) or anti-p53 antibody (clone DO7, DakoCytomation, Glostrup, Denmark, 1:50) in a humidified chamber for $60 \mathrm{~min}$ at room temperature. After washing, the slides were incubated with biotinylated rabbit anti-mouse IgG (Dako, Copenhagen, Denmark) at a dilution of 1:500, and detected with an ABC-peroxidase- Kit (Vector Laboratories, Burlingame, CA) with diaminobenzidine as the substrate, according to the manufacturer's protocol. Sections were counterstained with Mayer's hemalaun (Merck, Darmstadt, Germany), washed in tap water, and mounted using Aquatex (Merck). The specificity of 14-3-3 $\sigma$ antibody was assessed by blocking controls. Native 14-3-3 $\sigma$ protein (ProSci Incorporated, Poway, CA) was added to the primary antibody solution in tenfold excess $1 \mathrm{~h}$ before application onto tissue sections. Blocking of 14-3-3 $\sigma$ antibody resulted in no 14-3-3 $\sigma$ staining on 14-3-3 $\sigma$ positive tissue sections (not shown) demonstrating no cross reaction of this antibody with other members of the 14-3-3 protein family. Negative controls without primary antibody were included in each run.

Slices were evaluated by two independent pathologists (P.M. and K.S.) using light microscopy who had no prior knowledge of the clinical data. Antigen expression was defined as the presence of specific staining of cytoplasm of tumour cells for 14-3-3 $\sigma$ and nuclear staining for p53. 14-3-3 $\sigma$ and p53 overexpression was evaluated by calculating a total immunostaining score as the product of a proportion and intensity score. The proportion score described the estimated fraction of positive stained tumour cells $(0=$ none; $1=<10 \%$; $2=10-50 \% ; 3=51-80 \% ; 4=>80 \%$ ). The intensity score represented the estimated staining intensity ( 0 , no staining; 1 , weak; 2 , moderate; 3 , strong). The total score ranged from $0-12$. Regarding the total score the tissue samples were bimodally distributed with the nadir at a total score of 3-4. Therefore, 14-3-3 $\sigma$ "overexpression" was arbitrarily defined as a total score $>4$ as described previously [20].

\subsection{Statistical analysis}

Statistical analysis was performed using the Statistical Package of Social Science (SPSS, Version 16.0, Chicago IL). Descriptive statistics for continuous measures are given as the mean with the respective $95 \%$ confidence interval $(\mathrm{CI})$ in parenthesis. For discrete da- 
Table 1

Patients $(n=157)$ characteristics and clinicopathological features

\begin{tabular}{|c|c|c|c|}
\hline & & $\mathrm{n} /$ mean & $\% / \min -\max$ \\
\hline Age (years) & & 67.2 & $27-94$ \\
\hline \multirow[t]{2}{*}{ Gender } & female & 60 & 38.0 \\
\hline & male & 97 & 62.0 \\
\hline \multirow[t]{4}{*}{ Operative Procedure } & subtotal gastrectomy & 53 & 33.8 \\
\hline & total gastrectomy & 82 & 52.2 \\
\hline & resection of the gastric remnant & 13 & 8.3 \\
\hline & limited resection & 9 & 5.7 \\
\hline \multirow[t]{2}{*}{ Lymph node dissection } & D1 & 41 & 26.1 \\
\hline & $\mathrm{D} 2+\mathrm{D} 3$ & 116 & 73.9 \\
\hline \multirow[t]{4}{*}{ pT-category } & $\mathrm{pT} 1$ & 28 & 17.8 \\
\hline & 2 & 87 & 55.4 \\
\hline & 3 & 37 & 23.6 \\
\hline & 4 & 5 & 3.2 \\
\hline \multirow[t]{4}{*}{ pN-category } & pNO & 71 & 45.2 \\
\hline & 1 & 46 & 29.3 \\
\hline & 2 & 26 & 16.6 \\
\hline & 3 & 14 & 8.9 \\
\hline \multirow[t]{2}{*}{ M-category } & M0 & 146 & 93.0 \\
\hline & 1 & 11 & 7.0 \\
\hline \multirow[t]{5}{*}{ UICC-stage } & IA & 25 & 15.9 \\
\hline & IB & 34 & 21.7 \\
\hline & II & 43 & 27.4 \\
\hline & IIIA + IIIB & 33 & 21.0 \\
\hline & IV & 22 & 14.0 \\
\hline \multirow[t]{2}{*}{ R-Stage } & R0 & 150 & 95.5 \\
\hline & R1 & 7 & 4.5 \\
\hline \multirow[t]{2}{*}{ Tumor Differentiation } & G1-2 & 53 & 33.8 \\
\hline & G3-4 & 104 & 66.2 \\
\hline \multirow[t]{2}{*}{ Lauren Classification } & intestinal & 92 & 58.6 \\
\hline & diffuse & 65 & 41.4 \\
\hline \multirow[t]{2}{*}{ Chemotherapy } & neoadjuvant & 9 & 5.2 \\
\hline & adjuvant & 14 & 9.1 \\
\hline
\end{tabular}

min-max. . . lowest and highest value.

ta (e.g. 14-3-3 $\sigma$ expression) frequency counts and percentages were tabulated. Results of the various estimates were compared using either the $\chi^{2}$ test or Oneway ANOVA. Survival curves were calculated using the Kaplan and Meier method and compared by the logrank test. Follow-up time was censored if the patient was lost to follow-up. Patients who died without documented disease recurrence were considered censored for disease-free survival but were included as deaths for overall survival analysis. Factors shown to be of prognostic significance in univariate models were evaluated in a multivariate Cox regression model. $P<0.05$ was considered statistically significant.

\section{Results}

\subsection{Expression of 14-3-3 $\sigma$ and $p 53$}

Out of all cancer samples, 14-3-3 $\sigma$ was immunohistochemically detected in the cytoplasm of tumor cells in $122(77.7 \%)$ specimens whereas $35(22.3 \%)$ patients showed a negative staining reaction (Fig. 1). With re- gard to $14-3-3 \sigma$ positivity $(n=122), 65(53.3 \%)$ patients were with strong staining fraction (score 4), 50 $(41.0 \%)$ with moderate (score 3$)$, and $7(5.7 \%)$ with weak (score 2) staining contingent. According to the above mentioned criteria, 14-3-3 $\sigma$ was found to be overexpressed in 75 (47.8\%) of 157 cases. Adjacent normal epithelial cells were stained negatively or very weakly. Contrary, p53 immunostaining was detectable only in the nucleus of tumor cells (Fig. 2). Out of all cancer samples $67(42.7 \%)$ were immunopositive for p53, whereas $90(57.3 \%)$ lacked a positive staining reaction. Referring to immunopositivity, 49 (73.1\%) patients were with strong staining fraction (score 4), 7 (10.4\%) with moderate (score 3), and 11 (16.4\%) with weak (score 2) staining rate. The overexpression rate of p53 protein was $27.4 \%$. Adjacent normal epithelial cells were stained negatively for $\mathrm{p} 53$.

\subsection{Relationship between 14-3-3 $\sigma$ and p53 overexpression and clinicopathologic features}

14-3-3 $\sigma$ overexpression in carcinoma cells was statistically significantly associated with pT-stage $\left(\chi^{2}=\right.$ 


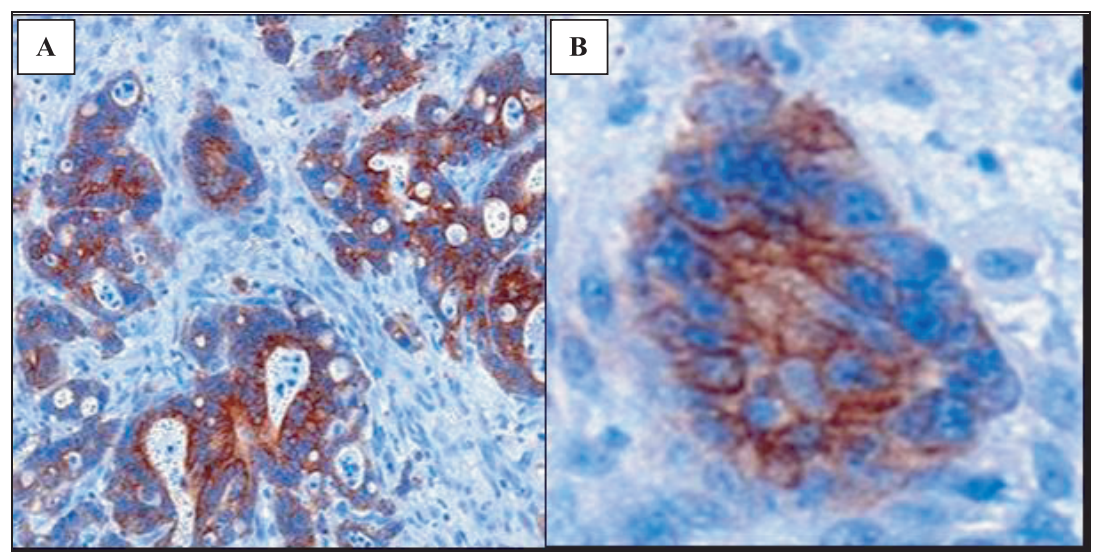

Fig. 1. Immunohistochemical staining for 14-3-3 $\sigma$ expression in human gastric cancer tissues with anti-14-3-3 $\sigma$ antibody, (A original magnification $\mathrm{x}$ 100; B original magnification $\mathrm{x} 400$ ). The examples show a strong specific cytoplasmic staining for 14-3-3 $\sigma$ and no staining in the nucleus and plasma membrane.

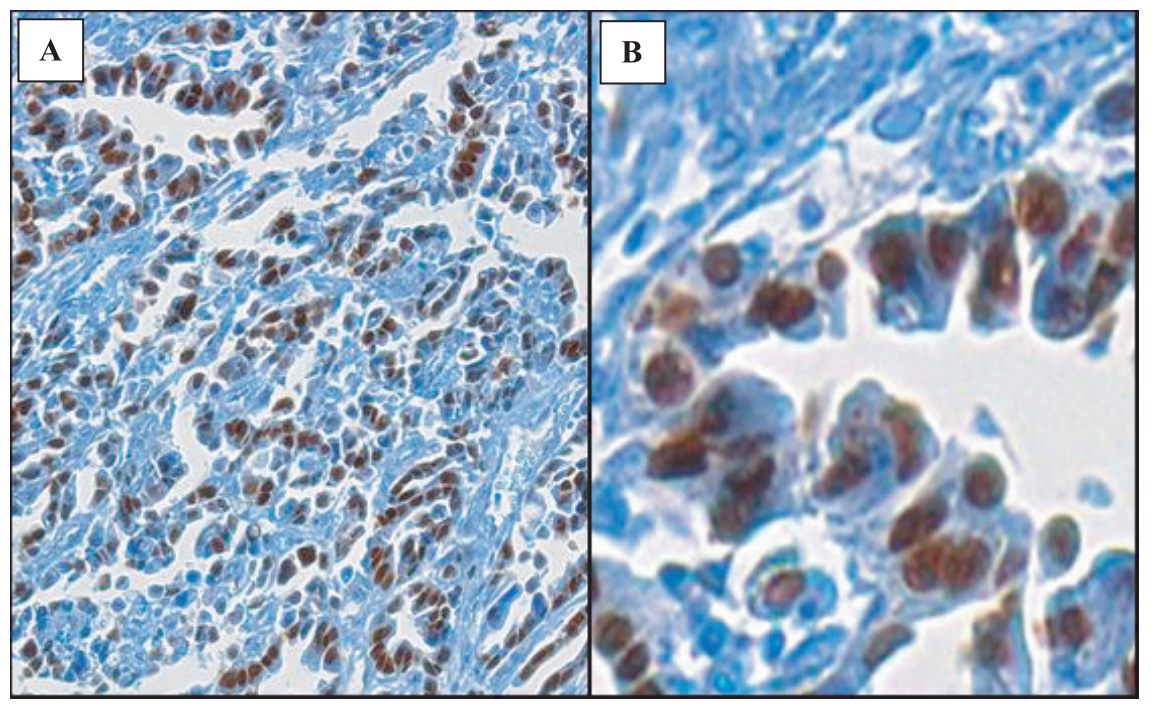

Fig. 2. Immunohistochemical staining for $\mathrm{p} 53$ expression in human gastric cancer tissues with anti-p53 antibody, (A original magnification $\mathrm{x}$ 100; B original magnification $\mathrm{x} 400$ ). The examples show a strong nuclear p53 staining.

$8.24 ; \mathrm{df}=3 ; p=0.041) \mathrm{pN}$-stage $\left(\chi^{2}=5.38 ; \mathrm{df}=\right.$ $1 ; p=0.015)$ and UICC-stage $\left(\chi^{2}=13.53 ; \mathrm{df}=5\right.$; $p=0.019)$ and showed a borderline significance with Lauren classification $\left(\chi^{2}=5.73 ; \mathrm{df}=2 ; p=0.057\right)$ (Table 2). No statistically significant association was found between p53 and common clinicopathologic features.

\subsection{Relationship between 14-3-3 $\sigma$ expression and p53 status}

Due to the fact that $14-3-3 \sigma$ is induced by $\mathrm{p} 53$ protein in response to $\gamma$ irradiation and other damaging agents and that 14-3-3 $\sigma$ has reciprocally influence on the p53 network we examined the relationship between 14-3-3 $\sigma$ and p53. Statistical analysis did not show significant association between the two investigated parameters.

\subsection{Survival analysis}

Regarding disease-free survival and overall survival, 14-3-3 $\sigma$ overexpression did not reveal statistical significance (Fig. 3). Univariate survival calculations for the total cohort revealed Lauren classification, tumor differentiation, depth (T-stage) of wall invasion, nodal status, presence of distant metastasis, UICC-stage, tu- 
Table 2

Relationship between 14-3-3 $\sigma$ overexpression and p53 overexpression and various clinicopathological parameters in 157 gastric cancer specimens

\begin{tabular}{|c|c|c|c|c|c|}
\hline & $\begin{array}{c}\text { All cases } \\
(n=75 ; 47.8 \%)\end{array}$ & $14-3-3 \sigma$ overexpression & $\begin{array}{c}\mathrm{p} * \\
(n=43 ; 27.4 \%)\end{array}$ & p53 overexpression & $\mathrm{p}^{*}$ \\
\hline \multicolumn{6}{|l|}{ Gender } \\
\hline male & 97 & $44(58.7)$ & n.s. & $29(67.4)$ & n.s. \\
\hline female & 57 & $31(41.3)$ & & $14(32.6)$ & \\
\hline \multicolumn{6}{|l|}{ Lauren classification } \\
\hline intestinal & 92 & $51(68.0)$ & 0.057 & $16(37.2)$ & n.s. \\
\hline diffuse & 65 & $24(32.0)$ & & $27(62.8)$ & \\
\hline \multicolumn{6}{|l|}{ Tumor differentiation } \\
\hline Grade $1 / 2$ & 53 & $29(38.7)$ & n.s. & $15(34.9)$ & n.s. \\
\hline Grade $3 / 4$ & 104 & $46(61.3)$ & & $28(65.1)$ & \\
\hline \multicolumn{6}{|l|}{ pT-category } \\
\hline pT1 & 28 & $7(9.3)$ & 0.041 & $9(20.9)$ & n.s. \\
\hline pT2 & 87 & $43(57.3)$ & & $22(51.2)$ & \\
\hline pT3 & 37 & $22(29.3)$ & & $10(23.3)$ & \\
\hline pT4 & 5 & $3(4.1)$ & & $2(4.7)$ & \\
\hline \multicolumn{6}{|l|}{ pN-category } \\
\hline N-pos & 79 & $45(60.0)$ & 0.015 & $25(58.1)$ & n.s. \\
\hline $\mathrm{N}$-neg & 78 & $30(40.0)$ & & $18(41.9)$ & \\
\hline \multicolumn{6}{|l|}{ M-category } \\
\hline M0 & 146 & $69(92.0)$ & n.s. & 38 (88.4) & n.s. \\
\hline M1 & 11 & $6(8.0)$ & & $5(11.6)$ & \\
\hline \multicolumn{6}{|l|}{ UICC stage } \\
\hline IA & 25 & $5(6.7)$ & 0.019 & 8 (18.6) & n.s. \\
\hline IB & 34 & $20(26.7)$ & & $10(23.3)$ & \\
\hline II & 43 & $19(25.3)$ & & $12(27.9)$ & \\
\hline IIIA + B & 33 & $18(24.0)$ & & $2(4.7)$ & \\
\hline IV & 22 & $13(17.3)$ & & $11(25.6)$ & \\
\hline
\end{tabular}

*Probability, P, from $\chi^{2}$ test.

Table 3

Prognostic factors examined in 157 adenocarcinomas of the stomach: an univariate approach to tumor relapse and cancer-specific mortality

\begin{tabular}{|c|c|c|c|c|}
\hline & \multicolumn{2}{|c|}{ DFS } & \multicolumn{2}{|c|}{ OS } \\
\hline & $\mathrm{X}^{2}$ & $\mathrm{P} *$ & $\mathrm{X}^{2}$ & $\mathrm{P} *$ \\
\hline Age $(\leqslant 70$ vs. $>70))$ & 0.001 & n.s. & 4.83 & 0.028 \\
\hline Lauren classification (intestinal vs. diffuse) & 6.01 & 0.049 & 2.58 & n.s. \\
\hline Operative procedure (subt. vs. total gastr. vs. gr vs. limited) & 2.35 & n.s. & 6.9 & n.s. \\
\hline Lymph node dissection (D1 vs. D2/3) & 1.76 & n.s. & 6.06 & 0.014 \\
\hline Tumor differentiation (G1/G2 vs. G3/G4) & 8.10 & 0.004 & 3.27 & n.s. \\
\hline pT-category (pT1 vs. pT2 vs. pT3 vs. pT4) & 31.80 & 0.001 & 22.36 & 0.001 \\
\hline pN-category $(\mathrm{pN}-$ vs. $\mathrm{pN}+)$ & 11.15 & 0.001 & 12.23 & 0.007 \\
\hline M-category (M0 vs. M1) & 18.03 & 0.001 & 21.54 & 0.001 \\
\hline UICC-stage (IA + IB vs. II + IIIA/B + IV) & 46.28 & 0.001 & 25.66 & 0.001 \\
\hline Residual disease (R0 vs. $\mathrm{R} 1+\mathrm{R} 2$ ) & 3.00 & n.s. & 0.59 & n.s. \\
\hline Anemia $(\leqslant 11 \mathrm{~g} \%$ vs. $>11 \mathrm{~g} \%))$ & 2.25 & n.s. & 1.89 & n.s. \\
\hline Tumor size $(\leqslant 4 \mathrm{~cm}$ vs. $>4 \mathrm{~cm}))$ & 12.43 & 0.001 & 1.67 & n.s. \\
\hline 14-3-3 $\sigma$ overexpression (yes vs. no) & 1.57 & n.s. & 0.55 & n.s. \\
\hline p53 overexpression (yes vs. no) & 2.50 & n.s. & 0.88 & n.s. \\
\hline $14-3-3 \sigma / \mathrm{p} 53$ overexpression (yes vs. no) & 4.91 & 0.027 & 0.23 & n.s. \\
\hline
\end{tabular}

DFS. . disease-free survival; OS. . overall survival;

* Probability, $P$, from $\chi^{2}$ test.

mor size and concomitant 14-3-3 $\sigma$ and p53 overexpression as significant predictors of disease-free survival (Fig. 4). Regarding overall survival, age, lymph node dissection, T stage, nodal status, presence of distant metastasis and UICC-stage were of prognostic sig- nificance, whereas gender and 14-3-3 $\sigma$ overexpression were not (Table 3).

In multivariate analysis, UICC stage was an independent prognostic factor for disease-free survival and overall survival (Table 4). 

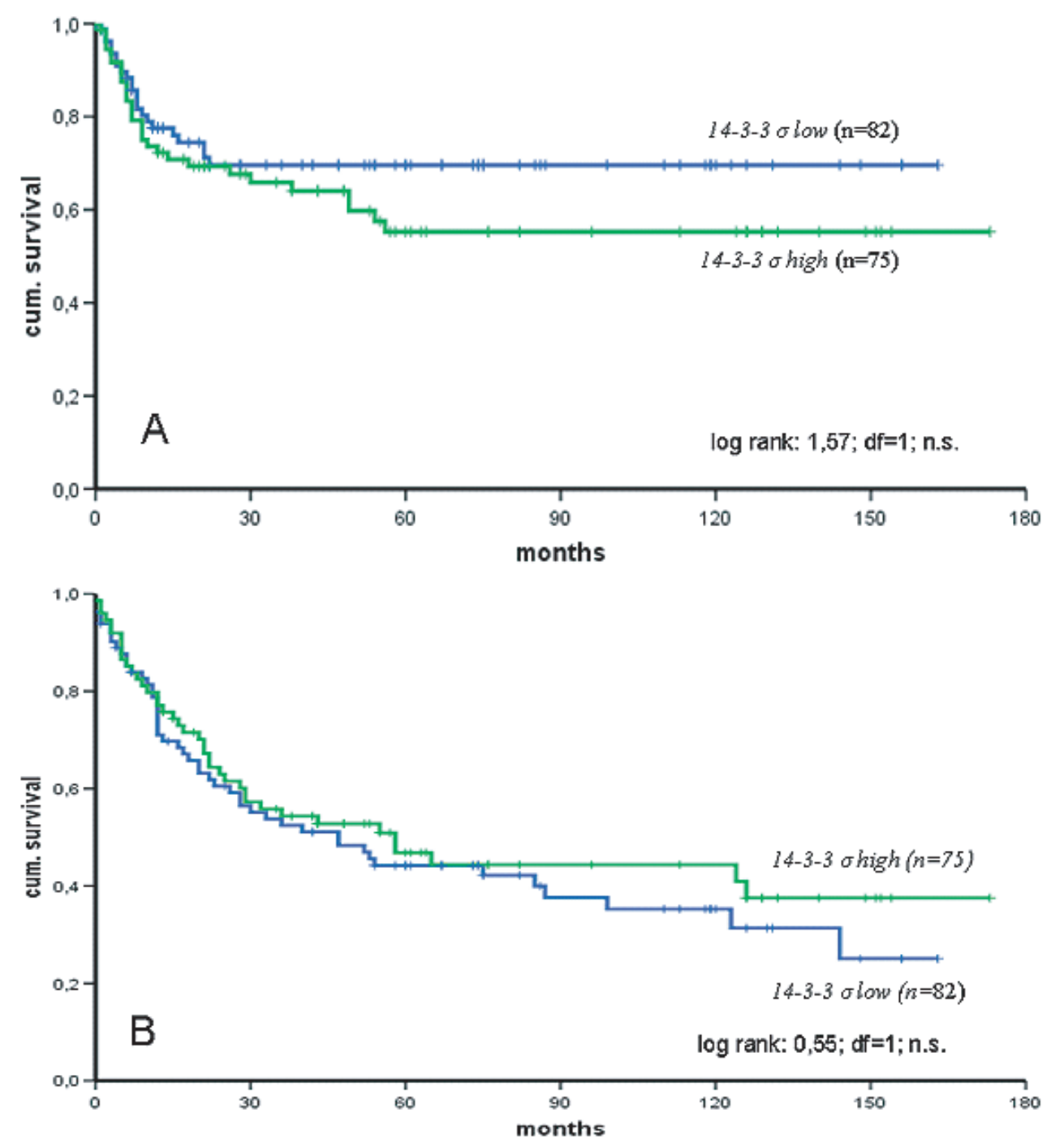

Fig. 3. Kaplan Meier survival curves with regard to disease-free-survival (A) and overall survival (B) of 157 patients with gastric carcinomas grouped according to their 14-3-3 $\sigma$ expression. high, patients with 14-3-3 $\sigma$ overexpressing tumor $(n=75)$. low, patients with tumor without 14-3-3 $\sigma$ overexpression $(n=82)$.

\section{Discussion}

14-3-3 proteins are a family of highly conserved cellular proteins that play key roles in the regulation of central physiological pathways. 14-3-3 $\sigma$, one of the seven $14-3-3$ genes, is said to hold an important role in human tumorigensis [21]. Several lines of evidence suggests that 14-3-3 $\sigma$ expression contributes to cancer development via the conference of apoptosis resistance, chemoresistance, and/or growth factor advantage to cancer cells, under certain growth conditions [22,23]. The present study revealed the expression of 14-3-3 $\sigma$ and its prognostic value in gastric cancer.
Recent studies have demonstrated that the loss of function of 14-3-3 $\sigma$ is either due to hypermethylation of the promoter or to induction of an oestrogenresponsive ubiquitin ligase that specifically targets 143-3 $\sigma$ for proteosomal degradation [6-12]. CpG islands hypermethylation and loss of 14-3-3 $\sigma$ expression was frequently found in different tumor entities such as hepatocellular carcinoma, lung cancer, prostate cancer, oral cancer, ovarian cancer or gastric cancer, whereas in colorectal cancer $\mathrm{CpG}$ islands were unmethylated [1016].

Investigations by Suzuki et al. revealed that 14-3-3 $\sigma$ methylation was frequently observed in poorly dif- 
Table 4

Multivariate analysis regarding disease-free and overall survival

\begin{tabular}{|c|c|c|c|c|}
\hline & \multicolumn{2}{|l|}{ DFS } & \multicolumn{2}{|l|}{ OS } \\
\hline & RHR $(95 \% \mathrm{CI})$ & $\mathrm{P}^{*}$ & RHR $(95 \% \mathrm{CI})$ & $\mathrm{P} *$ \\
\hline UICC-stage (II + IIIA/B + IV vs. IA + IB) & $5.91(1.99-16.80)$ & 0.000 & $2.59(2.26-11.95)$ & 0.000 \\
\hline 14-3-3 $\sigma$ overexpression (yes vs. no) & $0.59(0.33-1.86)$ & n.s. & $0.26(0.36-1.31)$ & n.s. \\
\hline
\end{tabular}

DFS. . disease- free survival; OS. . overall survival; RHR . . relative hazard ratio; CI. . confidence interval; * Probability, $P$, from $\chi^{2}$ test.

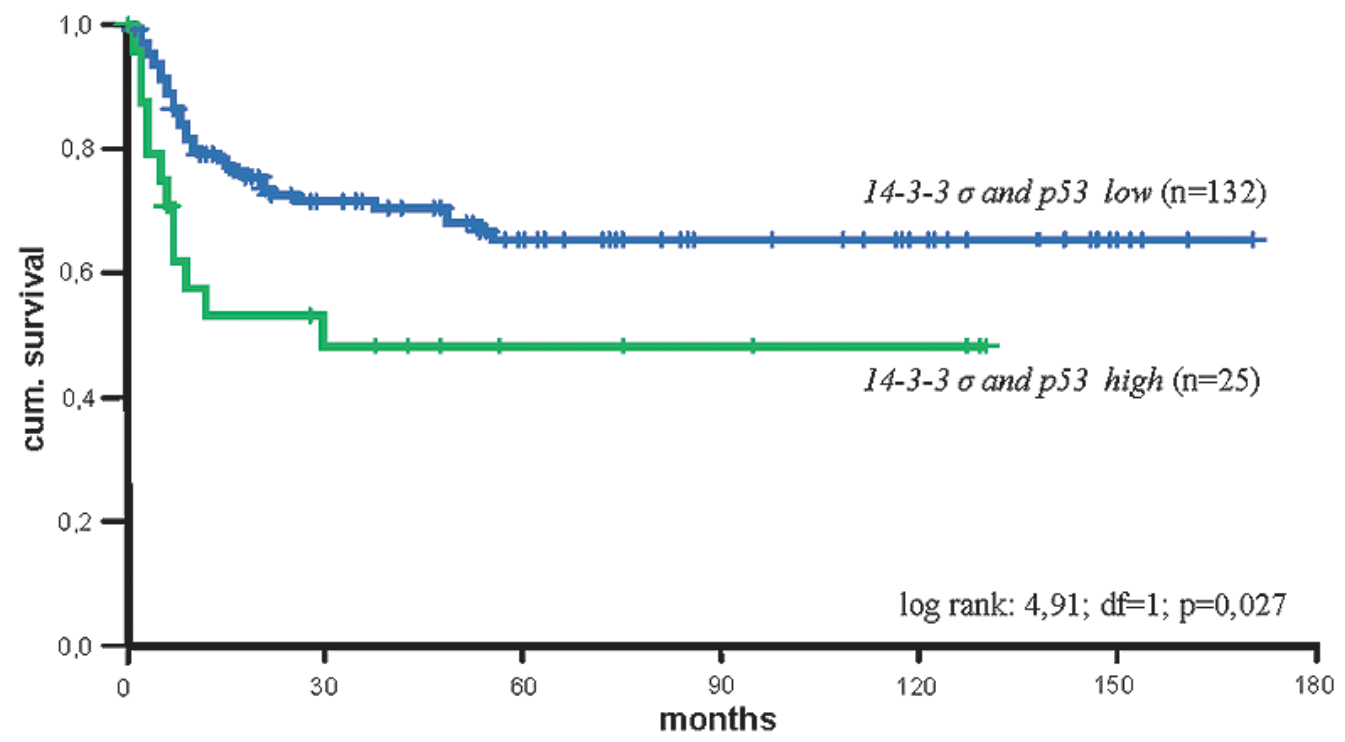

Fig. 4. Kaplan Meier survival curves with regard to disease-free-survival of 157 patients with gastric carcinomas with concomitant 14-3-3 $\sigma$ and p53 overexpression $(n=25)$. high, patients with concomitant 14-3-3 $\sigma$ and p53 overexpression $(n=25)$. low, patients without concomitant 14-3-3 $\sigma$ and p53 overexpression $(n=82)$.

ferentiated adenocarcinoma of the stomach suggesting that decreased expression of 14-3-3 $\sigma$ is associated with the developement of undifferentiated gastric cancer. In a series of 60 gastric cancer tissue samples they observed 14-3-3 $\sigma$ hypermethylation in $43 \%$, interpreting that the expression of 14-3-3 sigma is lost [11]. In contrast, other reports could show that 14-3-3 $\sigma \mathrm{mR}-$ NA and protein expression was strongly up-regulated in more than two thirds of gastrointestinal cancer patients [24]. This goes along with our findings where $77.7 \%$ of the study patients showed positivity for 143-3 $\sigma$ with an overexpression rate of $47.8 \%$. Tanaka et al. allready published data on 14-3-3 $\sigma$ expression in primary gastrointestinal malignancy. The analysis of the relationship between 14-3-3 $\sigma$ expression and common clinicopathologic factors in 34 gastric cancer patients revealed only the gross appearance as a significant parameter. Gastric cancer with Borrmann types 1 or 2 showed significantly higher 14-3-3 $\sigma$ expression in cancer cells than those with Borrmann types 3 or 4 [24]. The study population in this research is very small and a limiting factor for a clear conclusion. So we conducted an analysis with a larger study group to be able to make an improved statement. We found immunopositivity in $77.7 \%$ of all patients and out of these a high expression pattern in $74.2 \%$ of the cases. 14-3-3 $\sigma$ overexpression was statistically significantly associated with pT-stage, pN-stage, and UICC-stage, and showed a borderline significance with Lauren classification.

Contrary to other reported tumor entities, KaplanMeier survival analysis in this study showed that 14-3$3 \sigma$ overexpression does not predict survival in gastric cancer patients $[20,26]$. p53 mutation and thereby the loss of the suppressive function is a key event in half of all human cancers [27]. Since 14-3-3 $\sigma$ is integrated in the p53 network, dysregulation of 14-3-3 $\sigma$ may be jointly responsible for tumor development in p53 wild-type cancers. Nevertheless, most examinations on gastric cancer have not shown predictive or prognostic impact of p53 [28,29]. In the present study, we can confirm these findings. No correlation was found between p53 expression and prognosis. Interestingly, 
concomitant 14-3-3 $\sigma$ and p53 overexpression was extracted as a significant predictor of disease-free survival in univariate analysis.

Since the bigger part of the patients in this study did not receive perioperative chemotherapy or radiation treatment, the 14-3-3 $\sigma$ expression might not be influenced by therapy-induced DNA damage in vivo.

In conclusion, we demonstrated on a large study population with gastric cancer that 14-3-3 $\sigma$ is immunopositive in $77.7 \%$ of the patients. Concomitant 14-3-3 $\sigma$ and p53 overexpression in tumor cells of patients with gastric cancer identifies a population of patients with relatively unfavorable prognosis, however 14-3-3 $\sigma$ does not predict survival in gastric cancer patients like it does in other tumor entities.

\section{Conflict of interest}

No potential conflict of interest relevant to this article was reported.

\section{Acknowledgments}

The authors are grateful to Ms. Ines Tschörner for excellent technical assistance. Thanks go to Helmut Mühlböck from the "Tyrolean Tumorregister" for providing survival data.

Grant Support: Österreichische Krebshilfe-Krebsgesellschaft Tirol 2006.

\section{Author Contributions}

Conception and design: Gilbert Mühlmann, Albert Amberger.

Administrative support: Gilbert Mühlmann.

Provision of study materials or Patients: Hans Maier, Patrizia Moser, Kurt Werner Schmid, Matthias Zitt, Dietmar Öfner, Marion Zitt, Hannes M. Müller.

Data analysis and interpretation: Gilbert Mühlmann, Dietmar Öfner, Albert Amberger.

Manuscript writing: Gilbert Mühlmann, Albert Amberger.

Final approval of manuscript: Gilbert Mühlmann, Dietmar Öfner, Matthias Zitt, Hannes M. Müller, Hans Maier, Patrizia Moser, Kurt Werner Schmid, Marion Zitt, Albert Amberger.

\section{References}

[1] T.A. Chan, H. Hermeking, C. Lengauer, K.W. Kinzler and B. Vogelstein, 14-3-3Sigma is required to prevent mitotic catastrophe after DNA damage, Nature 401(6753) (7 Oct 1999), 616-620.

[2] H. Fu, R.R. Subramanian and S.C. Masters, 14-3-3 proteins: structure, function, and regulation, Annu Rev Pharmacol Toxicol 40 (2000), 617-647.

[3] E.W. Wilker, M.A. van Vugt, S.A. Artim et al., 14-3-3sigma controls mitotic translation to facilitate cytokinesis, Nature 446(7133) (15 Mar 2007), 329-332.

[4] H. Hermeking, C. Lengauer, K. Polyak et al., 14-3-3 sigma is a p53-regulated inhibitor of G2/M progression, Mol Cell 1(1) (Dec 1997), 3-11.

[5] H.Y. Yang, Y.Y. Wen, C.H. Chen, G. Lozano and M.H. Lee, 14-3-3 sigma positively regulates p53 and suppresses tumor growth, Mol Cell Biol 23(20) (Oct 2003), 7096-7107.

[6] C.B. Umbricht, E. Evron, E. Gabrielson, A. Ferguson, J. Marks and S. Sukumar, Hypermethylation of 14-3-3 sigma (stratifin) is an early event in breast cancer, Oncogene 20(26) (7 Jun 2001), 3348-3353.

[7] M. Gasco, A. Sullivan, C. Repellin et al., Coincident inactivation of 14-3-3sigma and p16INK4a is an early event in vulval squamous neoplasia, Oncogene 21(12) (14 Mar 2002), 1876-1881.

[8] A.S. Vercoutter-Edouart, J. Lemoine, X. Le Bourhis et al., Proteomic analysis reveals that 14-3-3sigma is down-regulated in human breast cancer cells, Cancer Res 61(1) (1 Jan 2001), 76-80.

[9] T. Urano, T. Saito, T. Tsukui et al., Efp targets 14-3-3 sigma for proteolysis and promotes breast tumour growth, Nature 417(6891) (20 Jun 2002), 871-875.

[10] N. Iwata, H. Yamamoto, S. Sasaki et al., Frequent hypermethylation of CpG islands and loss of expression of the 14-33 sigma gene in human hepatocellular carcinoma, Oncogene 19(46) (2 Nov 2000), 5298-302.

[11] H. Suzuki, F. Itoh, M. Toyota, T. Kikuchi, H. Kakiuchi and K. Imai, Inactivation of the 14-3-3 sigma gene is associated with 5' $\mathrm{CpG}$ island hypermethylation in human cancers, Cancer Res 60(16) (15 Aug 2000), 4353-4357.

[12] M. Kaneuchi, M. Sasaki, Y. Tanaka et al., Expression and methylation status of 14-3-3 sigma gene can characterize the different histological features of ovarian cancer, Biochem Biophys Res Commun 316(4) (16 Apr 2004), 1156-1162.

[13] H. Osada, Y. Tatematsu, Y. Yatabe et al., Frequent and histological type-specific inactivation of 14-3-3sigma in human lung cancers, Oncogene 21(15) (4 Apr 2002), 2418-2424.

[14] L. Cheng, C.X. Pan, J.T. Zhang et al., Loss of 14-3-3sigma in prostate cancer and its precursors, Clin Cancer Res 10(9) (1 May 2004), 3064-3068.

[15] M. Gasco, A.K. Bell, V. Heath et al., Epigenetic inactivation of 14-3-3 sigma in oral carcinoma: association with p16(INK4a) silencing and human papillomavirus negativity, Cancer Res 62(7) (1 Apr 2002), 2072-2076.

[16] M. Ide, T. Nakajima, T. Asao and H. Kuwano, Inactivation of 14-3-3 sigma by hypermethylation is a rare event in colorectal cancers and its expression may correlate with cell cycle maintenance at the invasion front, Cancer Lett 207(2) (30 Apr 2004), 241-249.

[17] C.H. Wittekind, TNM Atlas: Illustrated Guide to the TNM Classification of Malignant Tumours (UICC). WileyBlackwell. ISBN: 9780471743019. 
[18] WHO Classification of Tumors. Pathology and Genetics of Tumors of Digestive System. IARC Press 2000.

[19] J.S. Macdonald, S.R. Smalley, J. Benedetti et al., Chemoradiotherapy after surgery compared with surgery alone for adenocarcinoma of the stomach or gastroesophageal junction, $N$ Engl J Med 345 (2001), 725-730.

[20] A. Perathoner, D. Pirkebner, G. Brandacher et al., 14-3-3sigma expression is an independent prognostic parameter for poor survival in colorectal carcinoma patients, Clin Cancer Res 11(9) (1 May 2005), 3274-3279.

[21] H. Hermeking, 14-3-3 proteins and cancer biology, Semin Cancer Biol 16(3) (Jun 2006), 161

[22] T. Samuel, H.O. Weber, P. Rauch, B. Verdoodt, J.T. Eppel, A. McShea et al., The G2/M regulator 14-3-3sigma prevents apoptosis through sequestration of Bax, J Biol Chem 276 (2001), 45201-45206.

[23] Y. Zhang, M. Karas, H. Zhao H, S. Yakar and D. LeRoith, 14-33 sigma mediation of cell cycle progression is p53-independent in response to insulin-like growth factor-I receptor activation, J Biol Chem 279 (2004), 34353-34360.
[24] K. Tanaka, T. Hatada, M. Kobayashi et al., The clinical implication of 14-3-3 sigma expression in primary gastrointestinal malignancy, Int J Oncol 25(6) (Dec 2004), 1591-1597.

[25] K. Laimer, N. Blassnig, G. Spizzo et al., Prognostic significance of 14-3-3sigma expression in oral squamous cell carcinoma (OSCC), Oral Oncol 45(2) (Feb 2009), 127-134.

[26] N.K. Yoon, D.B. Seligson, D. Chia et al., Higher expression levels of 14-3-3sigma in ductal carcinoma in situ of the breast predict poorer outcome, Cancer Biomark 5(4) (2009), 215-24.

[27] J.M. Nigro, S.J. Baker, A.C. Preisinger et al., Mutations in the p53 gene occur in diverse human tumour types, Nature 342(6250) (7 Dec 1989), 705-708.

[28] H.H. Ishii, G.C. Gobe and Y. Ebihara, p53 is an indicator of tumor progression in early but not advanced gastric carcinomas, Hepatogastroenterology 54(79) (Oct-Nov 2007), 2159-2163.

[29] U. Drebber, S.E. Baldus, B. Nolden et al., The overexpression of c-met as a prognostic indicator for gastric carcinoma compared to p53 and p21 nuclear accumulation, Oncol Rep 19(6) (Jun 2008), 1477-1483. 


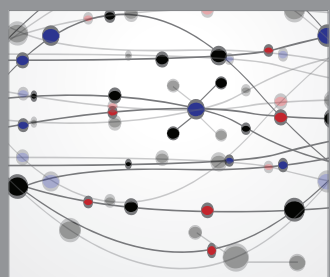

The Scientific World Journal
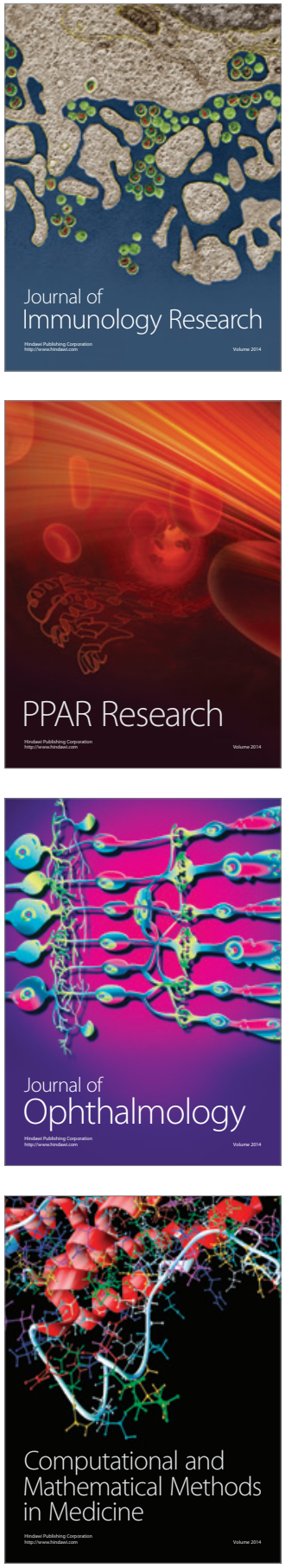

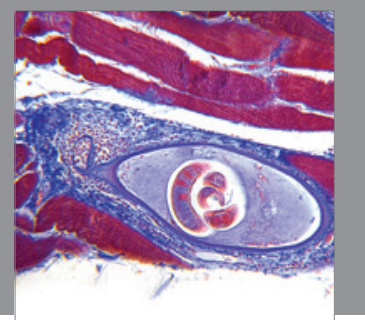

Gastroenterology

Research and Practice
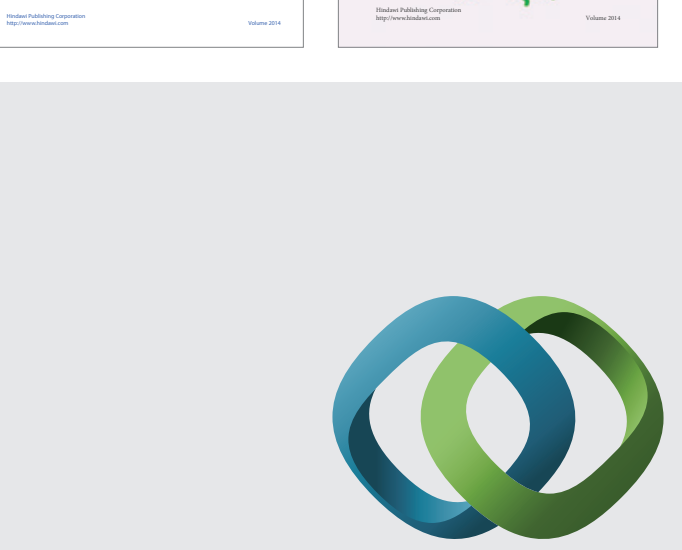

\section{Hindawi}

Submit your manuscripts at

http://www.hindawi.com
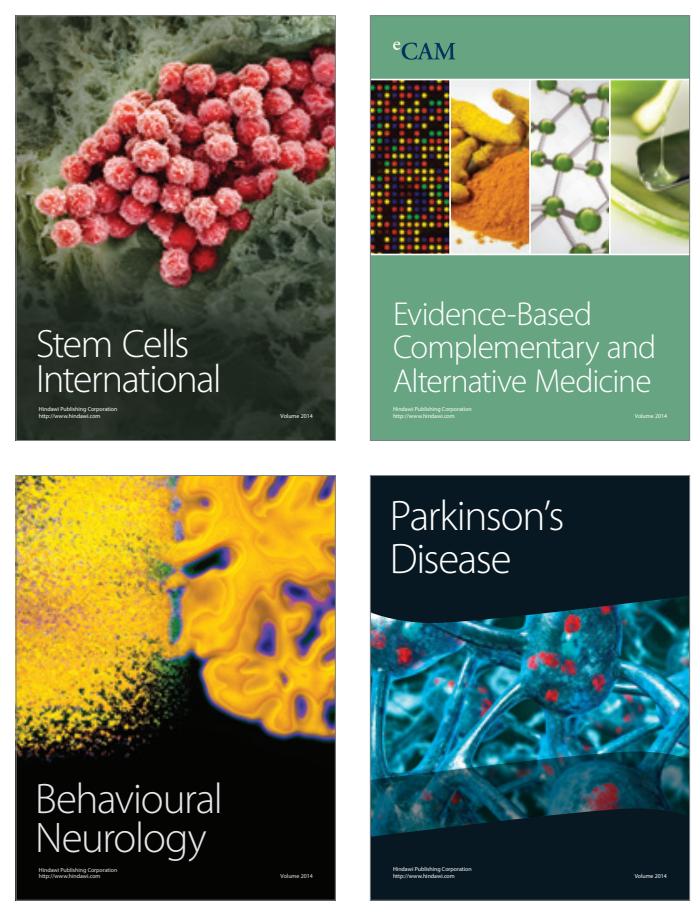

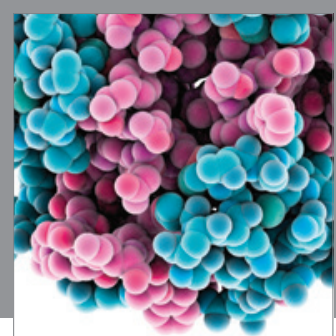

Journal of
Diabetes Research

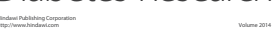

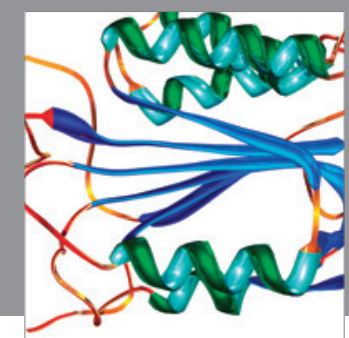

Disease Markers
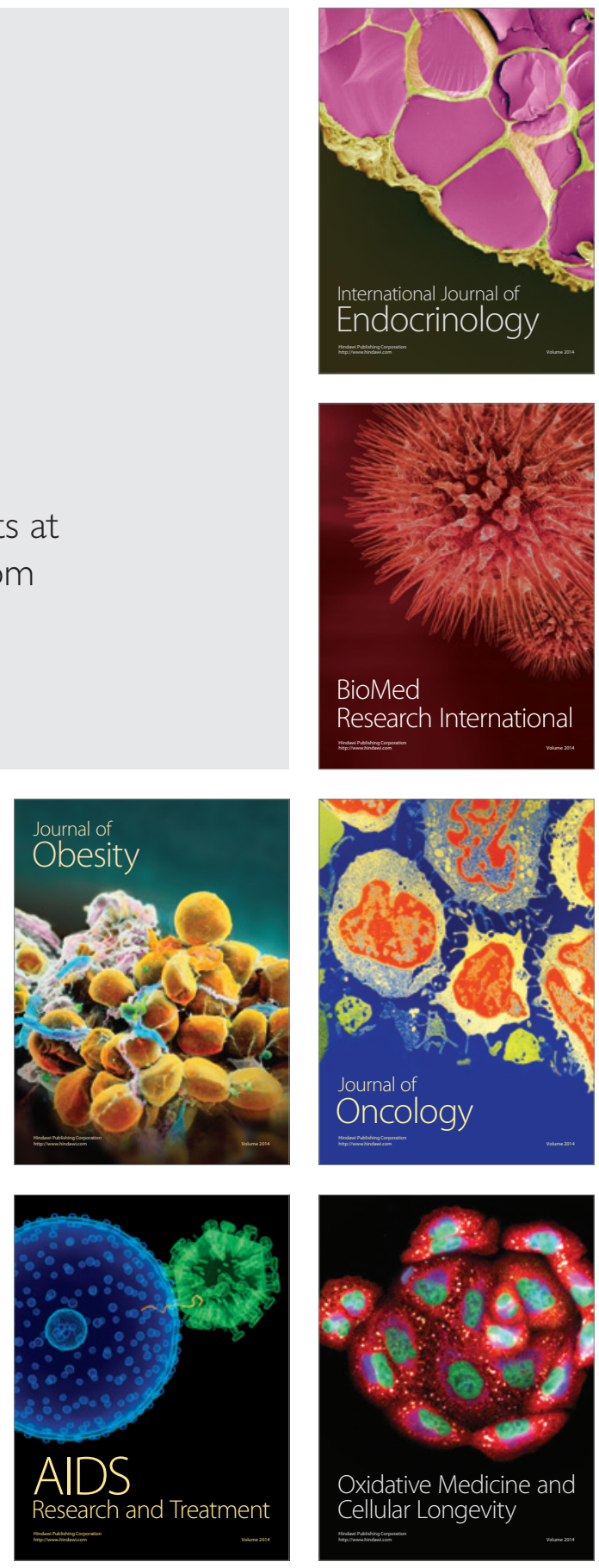